DOI: 10.12957/demetra.2018.32906

\title{
Inadequação da rotulagem de alimentos alergênicos: risco para indivíduos com hipersensibilidade alimentar
}

\author{
Inadequate labeling of allergenic food: the risk for individuals with food hypersensitivity
}

\author{
Camila Cristina Santos de Miranda ${ }^{1}$ \\ Larissa Lovatto Amorin Gama' \\ 1 Centro Universitário de Belo Horizonte, Instituto \\ de Ciências Biológicas e Saúde, Curso de \\ Nutrição. Belo Horizonte, MG, Brasil. \\ Correspondência / Correspondence \\ Larissa Lovatto Amorin Gama \\ E-mail: lovatto.larissa@gmail.com
}

\section{Resumo}

Objetivo: Analisar a adequação da rotulagem de alimentos alergênicos de acordo com a Resolução n.o 26, de 02 de julho de 2015. Metodologia: Verificou-se a concordância das declarações de alérgenos em 221 rótulos de alimentos industrializados comercializados em três redes distintas de supermercados. Os produtos foram subdivididos em dez grupos de alimentos (pães e cereais; peixes e crustáceos; ovos e derivados; sementes oleaginosas; leites e derivados; soja e derivados; massas; doces, bolos e biscoitos; cárneos e embutidos e bebidas alcoólicas) e foram categorizados como "inadequados" quando descumpriram qualquer critério estabelecido na resolução. Para detalhar as inadequações, foram adotadas as seguintes classificações: localização incorreta da declaração no rótulo, declaração incompleta e/ou formatação inadequada e declaração inexistente. Resultados: Do total de rótulos avaliados, 31,7\% $(\mathrm{n}=70)$ foram identificados como inadequados. Destas inadequações, observouse que $48,6 \%$ foram classificados como "Declaração inexistente". Observou-se, ainda, que foram encontradas inadequações nos dez grupos de alimentos avaliados. Conclusão: As normas regulamentadoras nacionais de rotulagem dispõem inovações na perspectiva de defesa do consumidor alérgico; no entanto, ainda se observam diversas irregularidades no seu cumprimento. Investigações desta natureza são relevantes para a saúde pública e devem ser realizadas para exigir a fiscalização e implementação das legislações vigentes.

Palavras-chave: Rotulagem de alimentos. Hipersensibilidade alimentar. Legislação sobre alimentos. 


\section{Abstract}

Objective: To analyze labeling adequacy of allergenic foods in accordance with Resolution no. 26, of July 2, 2015. Methodology: The agreement of allergen declarations was evaluated on 221 labels of processed foods commercialized in three distinct supermarket chains. The products were subdivided into ten food groups (breads and cereals; fish and crustaceans; eggs and derivatives; oily seeds; milk and derivatives; soybeans and derivatives; pastas; sweets, cakes and biscuits; meats and processed meats and alcoholic beverages) and were categorized as "inadequate" when they failed to meet any criteria set forth in the resolution. In order to detail the inadequacies, the following classifications were adopted: incorrect location of the declaration on the label, incomplete declaration and/or inadequate formatting and nonexistent declaration. Results: The evaluated food labels showed that $31.7 \%(n=70)$ were identified as inadequate. Of these inadequacies, it was observed that $48.6 \%$ were classified as "Nonexistent Declaration". It was also observed that the inadequacies were found in the ten food groups. Conclusion: National regulatory labeling norms have innovated regarding the protection of allergic consumers, however, there are still several irregularities in their compliance. Investigations of this nature are relevant to public health and must be carried out to demand supervision and implementation of the current legislation.

Keywords: Food labeling. Food hypersensitivity. Legislation. Food.

\section{Introdução}

A rotulagem de alimentos tornou-se obrigatória no Brasil com a criação da Agência Nacional de Vigilância Sanitária (ANVISA), em 1999. Este órgão estabelece os informes necessários aos rótulos para assegurar a qualidade dos produtos com informações adequadas para os consumidores. ${ }^{1,2}$

De acordo com a Resolução da Diretoria Colegiada (RDC) n.o 259/2002, que aprova o regulamento técnico para rotulagem de alimentos embalados, rotulagem é toda inscrição, legenda, imagem ou toda matéria descritiva ou gráfica, escrita, impressa, gravada em relevo ou litografada ou colada sobre a embalagem do alimento. Ainda, o rótulo é uma das ferramentas de comunicação entre as propriedades nutricionais de um produto e seus consumidores, sendo assim um elemento importante para a saúde pública., 
À vista disso, a rotulagem é um recurso que auxilia na orientação de hábitos alimentares, como também na prevenção de reações adversas associadas diretamente ao alimento, como as alergias e intolerâncias alimentares. ${ }^{5,6} \mathrm{Em}$ indivíduos alérgicos a alimentos, até mesmo quantidades mínimas ingeridas são suficientes para desencadear reações graves. ${ }^{7}$ As principais manifestações clínicas de uma alergia alimentar compreendem reações mucocutâneas (urticária, angiodema, eritema, prurido), no trato gastrointestinal (diarreia, dor abdominal, náuseas, vômitos), no sistema respiratório (esternutação, rinorreia, tosse, dispneia, pieira) e cardiovascular (atordoamento, hipotensão, síncope). No entanto, a maior preocupação em uma crise alérgica é a reação anafilática, uma manifestação súbita e grave que pode ser fatal se não for tratada imediatamente. ${ }^{8}$

Vale ressaltar que o número de casos de alergias alimentares tem aumentado notavelmente na última década em todo o mundo. ${ }^{9}$ Nos Estados Unidos, sua prevalência aumentou $18 \% \mathrm{em} \mathrm{dez}$ anos na população pediátrica; na Europa, 17 milhões de indivíduos têm algum tipo de alergia alimentar. ${ }^{710}$ Dados recentes da Associação Brasileira de Alergia e Imunologia (ASBAI) ${ }^{11}$ estimam que 10 milhões de brasileiros sejam afligidos por alergias alimentares, o que equivale a $5 \%$ da população.

As reações de hipersensibilidade podem ser ocasionadas por numerosos alimentos, mas aproximadamente $90 \%$ dos casos relatados de alergia alimentar são induzidos por oito alimentos principais: ovo, trigo, soja, amendoim, castanha, leite, peixe e crustáceo. ${ }^{7}$ Vale ressaltar que o Codex Alimentarius, organismo da Food and Agriculture Organization (FAO), reconhece esses alimentos como alergênicos de alta relevância para a saúde pública. ${ }^{12,13}$

No Brasil, a regulamentação sobre a rotulagem de alimentos alergênicos é recente. Em 3 de julho de 2015, foi publicada a RDC n. 26 , que dispõe sobre os requisitos para rotulagem obrigatória dos principais alimentos que causam alergias alimentares. A partir desta data, as empresas obtiveram o prazo de 12 meses para as adequações obrigatórias nos produtos abrangidos por esta resolução, incluindo alimentos, bebidas, ingredientes, aditivos alimentares e coadjuvantes da tecnologia embalados na ausência dos consumidores. ${ }^{14}$ Nessa perspectiva, o esclarecimento adequado da rotulagem ao consumidor alérgico é fundamental, pois este poderá valer-se de informações completas que impedirão o consumo de alimentos que poderão desencadear possíveis reações adversas. ${ }^{715}$

Como uma das estratégias do tratamento das alergias alimentares consiste na exclusão do alimento alergênico da dieta, ${ }^{16} \mathrm{o}$ acesso às informações adequadas, confiáveis e claras referentes à rotulagem é imprescindível para evitar e controlar o risco de reações de hipersensibilidade alimentar. ${ }^{12}$ Nesse sentido, o presente estudo teve como objetivo analisar a adequação da rotulagem de alimentos alergênicos de acordo com a Resolução n.. 26/2015. 


\section{Metodologia}

Trata-se de estudo de caráter avaliativo e de abordagem quantitativa que verificou a concordância das declarações de alérgenos em 221 rótulos de alimentos industrializados comercializados em três redes distintas de supermercados, situadas nos municípios de Belo Horizonte e Sabará - MG, no período de agosto a outubro de 2016.

Os rótulos dos produtos de diversas marcas foram analisados sob as disposições da RDC n.. 26/2015 e categorizados como "inadequados" quando descumpriram qualquer critério estabelecido na resolução. Desta maneira, os alimentos que contêm ou são derivados dos alimentos alergênicos listados na referida resolução, de acordo com o artigo 6o-, devem trazer a seguinte declaração obrigatória: "ALÉRGICOS: CONTÉM (NOMES COMUNS DOS ALIMENTOS QUE CAUSAM ALERGIAS ALIMENTARES), ALÉRGICOS: CONTÉM DERIVADOS DE (NOMES COMUNS DOS ALIMENTOS QUE CAUSAM ALERGIAS ALIMENTARES) OU ALÉRGICOS: CONTÉM (NOMES COMUNS DOS ALIMENTOS QUE CAUSAM ALERGIAS ALIMENTARES) E DERIVADOS", conforme o caso.

Quando não for possível garantir a ausência de contaminação cruzada por alérgenos alimentares, segundo o artigo 7ō, deve constar no rótulo a declaração: "ALÉRGICOS: PODE CONTER (NOMES COMUNS DOS ALIMENTOS QUE CAUSAM ALERGIAS ALIMENTARES)", conforme o caso. Ainda, estas advertências devem estar legíveis, localizadas imediatamente após ou abaixo da lista de ingredientes e com os requisitos de formatação na declaração: caixa alta, negrito, cor contraste com o fundo do rótulo e altura igual ou superior à altura da letra utilizada na lista de ingredientes. ${ }^{14}$

Sendo assim, as inadequações foram classificadas conforme especificado no quadro 1.

Quadro 1. Classificação das inadequações verificadas nos rótulos dos produtos analisados, Belo Horizonte e Sabará - MG, 2016.

\begin{tabular}{|ll|}
\hline \multicolumn{1}{|c|}{ Inadequação } & \multicolumn{1}{c|}{ Classificação } \\
\hline Declaração inexistente & $\begin{array}{l}\text { Rótulos que não apresentaram a declaração obrigatória de } \\
\text { ingredientes alergênicos. }\end{array}$ \\
\hline Declaração incompleta & Rótulos que omitiram o termo "AlérGicos". \\
\hline $\begin{array}{l}\text { Localização incorreta da } \\
\text { declaração }\end{array}$ & $\begin{array}{l}\text { Rótulos em que a informação da presença de alergênicos } \\
\text { não se encontrava logo após a lista de ingredientes. }\end{array}$ \\
\hline $\begin{array}{l}\text { Formatação inadequada } \\
\text { da declaração }\end{array}$ & $\begin{array}{l}\text { Rótulos que não estavam de acordo com a formatação } \\
\text { estabelecida na resolução. }\end{array}$ \\
\hline
\end{tabular}


Como critérios de inclusão, foram selecionados os produtos comercializados a partir do dia três de julho de 2016 que poderiam conter qualquer um dos principais alérgenos alimentares explicitados no anexo da referida resolução (trigo, cevada, centeio, aveia; crustáceos; ovos; peixes; amendoim; soja; leite de animais mamíferos; amêndoa; avelãs; castanha-de-caju; castanha-do-brasil; macadâmias; nozes; pecãs; pistaches; castanhas e látex natural). Esta data representou o final do prazo de 12 meses estabelecido pela resolução para que as indústrias realizassem as adequações necessárias na rotulagem de seus produtos.

Os critérios de exclusão compreenderam os alimentos comercializados sem embalagem, aqueles embalados nos pontos de venda a pedido do consumidor e os embalados, preparados ou fracionados nos serviços de alimentação e comercializados no próprio estabelecimento.

Os produtos foram selecionados aleatoriamente nos estabelecimentos comerciais, seguidos de registros fotográficos das informações pertinentes para avaliação. Para fins de categorização, os produtos foram subdivididos em dez grupos de alimentos: pães e cereais; peixes e crustáceos; ovos e derivados; sementes oleaginosas; leites e derivados; soja e derivados; massas; doces, bolos e biscoitos; cárneos e embutidos e as bebidas alcoólicas. Os dados foram transcritos para uma tabela com o auxílio do software Microsoft Office Excel 2010, e para a análise descritiva, foi adotado o software estatístico IBM SPSS Statistics (Statistical Package for Social Sciences), versão 19 (Chicago, Illinois, EUA).

\section{Resultados}

Dos 221 rótulos analisados, 38,5\% $(\mathrm{n}=85)$ foram selecionados no supermercado A, 33,9\% $(n=75)$ no B e 27,6\% (n=61) no estabelecimento C. Em relação aos grupos de alimentos, 33,0\% $(\mathrm{n}=73)$ corresponderam ao grupo de leites e derivados; $13,6 \%(\mathrm{n}=30)$ ao grupo de pães e cereais; 12,2\% ( $\mathrm{n}=27)$ a doces, bolos e biscoitos; $10,0 \%(\mathrm{n}=22)$ a cárneos e embutidos; 7,3\% $(\mathrm{n}=16)$ a ovos e derivados; $6,3 \%(\mathrm{n}=14)$ a massas; $4,5 \%(\mathrm{n}=10)$ a peixes e crustáceos; $4,5 \%(\mathrm{n}=10)$ a sementes oleaginosas; 4,5\% $(\mathrm{n}=10)$ a bebidas alcoólicas e 4,1\% $(\mathrm{n}=9)$ para produtos de soja e derivados.

Do total de rótulos avaliados, 31,7\% $(n=70)$ foram classificados como inadequados. A figura 1 apresenta as classificações das inadequações registradas. 


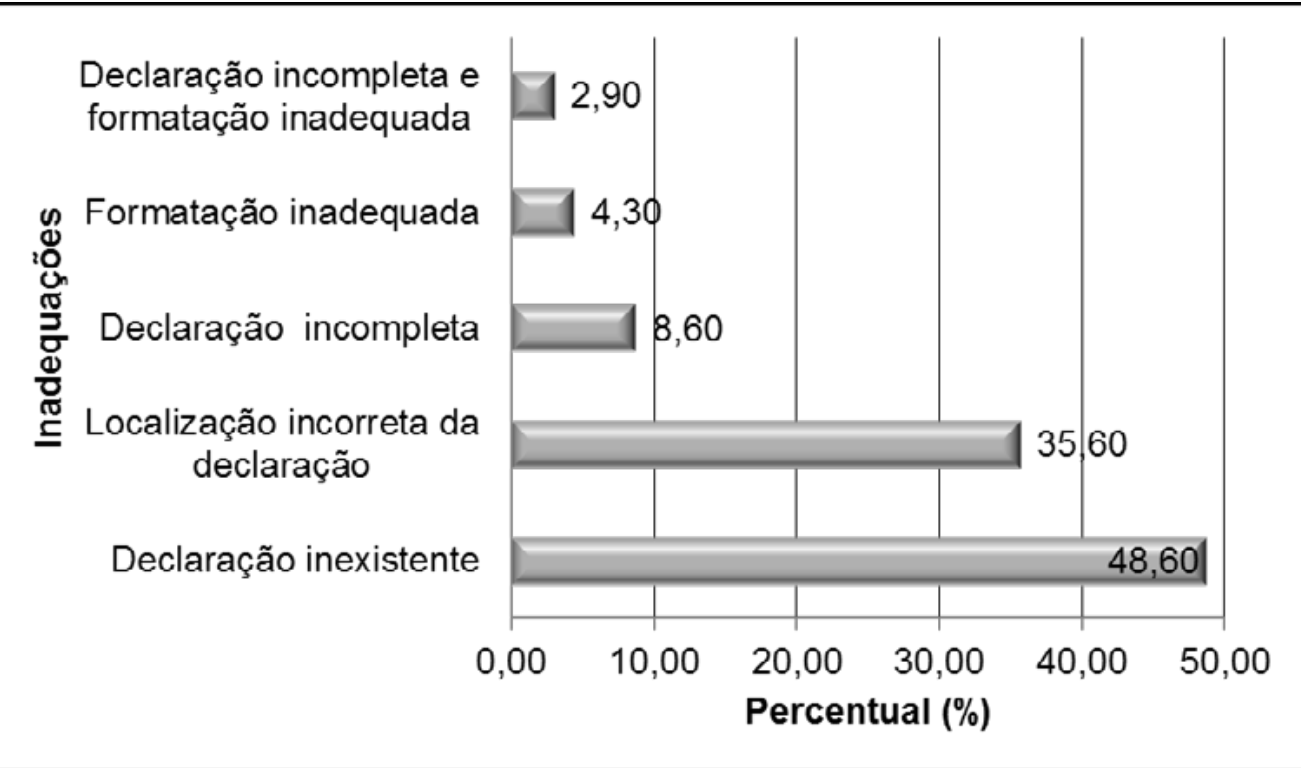

Figura 1. Inadequações verificadas nas análises da informação de alergênicos nos rótulos de produtos comercializados em diferentes estabelecimentos (\%), 2016.

Observa-se que a ausência de declaração de alergênicos no rótulo $(48,60 \%)$ foi a inadequação mais encontrada. As inconformidades observadas neste estudo podem dificultar e/ou limitar a identificação da presença de ingredientes que devem ter seu consumo restringido por indivíduos com hipersensibilidade alimentar. As adequações e inadequações segundo os grupos de alimentos analisados encontram-se na tabela 1 . 
Tabela 1. Avaliação da adequação dos rótulos dos produtos por grupos de alimentos, (\%, n). Belo Horizonte e Sabará - MG, 2016.

\begin{tabular}{lcc}
\hline Grupo de alimentos & $\begin{array}{c}\text { Rótulos } \\
\text { Inadequados* } \\
(\mathrm{n})\end{array}$ & $\begin{array}{c}\text { Rótulos } \\
\text { Adequados* } \\
\%(\mathrm{n})\end{array}$ \\
\hline Peixes e crustáceos & $4,3(3)$ & $4,6(7)$ \\
Soja e derivados & $4,3(3)$ & $4,0(6)$ \\
Cárneos e embutidos & $5,7(4)$ & $12,6(19)$ \\
Bebidas alcoólicas & $5,7(4)$ & $4,0(6)$ \\
Pães e cereais & $7,1(5)$ & $16,6(25)$ \\
Ovos e derivados & $7,1(5)$ & $7,3(11)$ \\
Sementes oleaginosas & $7,1(5)$ & $3,3(5)$ \\
Massas & $10,0(7)$ & $4,6(7)$ \\
Doces, bolos e biscoitos & $14,3(10)$ & $10,6(16)$ \\
Leites e derivados & $34,4(24)$ & $32,4(49)$ \\
\hline Total & $100(70)$ & $100(151)$ \\
\hline
\end{tabular}

* Rótulos analisados de acordo com a RDC n. ${ }^{\circ}$ 26, de 02 de julho de 2015.

Nota-se que as inadequações foram verificadas em todos os grupos de alimentos, o que implica maior risco para os consumidores alérgicos.

De acordo com a tabela 2, que apresenta as inadequações detalhadas para os dez grupos de alimentos, nota-se que nove grupos apresentaram a classificação "Declaração inexistente", evidenciando a negligência por parte das empresas que comercializam os mais variados tipos de produtos alimentares com ingredientes alergênicos. O grupo "Pães e cereais" se destacou por apresentar a declaração de alérgenos em todos os rótulos avaliados, mas eles se mostraram inadequados em pelo menos um dos quesitos investigados. Já o grupo "Soja e derivados" não declarou a presença de ingredientes alergênicos em nenhum rótulo avaliado. 


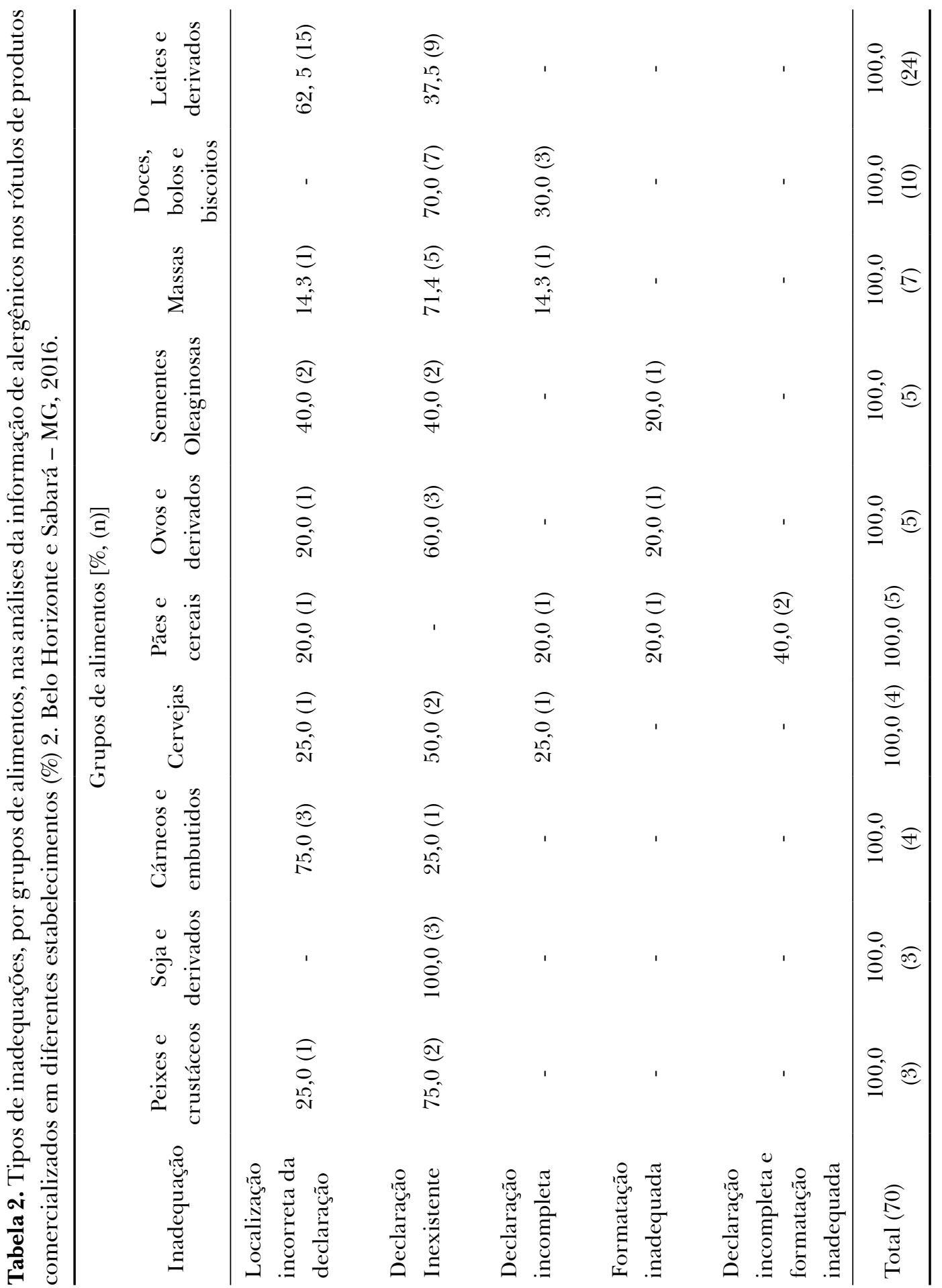




\section{Discussão}

Os rótulos analisados neste estudo apresentaram inadequações em relação à recente regulamentação para a rotulagem de produtos contendo ingredientes alergênicos. Jardim et al. ${ }^{17}$ afirmaram que muitas indústrias de alimentos em geral não seguem determinados requisitos legais vigentes que regem a rotulagem. Nesse sentido, torna-se imperativo que as indústrias alimentícias proporcionem o acesso às informações seguras e úteis sobre o produto que estão fornecendo aos consumidores e que atendam às exigências dos regulamentos, ${ }^{18}$ principalmente para os consumidores sensíveis a alérgenos alimentares. Uma vez que tais recomendações são descumpridas pelas empresas, as penalidades constituídas em termos de infração sanitária, sem prejuízo das responsabilidades civil, administrativa e penal cabíveis, podem ser instauradas. ${ }^{14}$

Para a aprovação desta nova resolução nacional, foi necessária a reivindicação de instituições, como a ASBAI e a Campanha "Põe no Rótulo". Este movimento nas mídias sociais, formado por indivíduos alérgicos a alimentos e seus familiares, permitiu a exposição dos desafios para a interpretação dos rótulos. As principais dificuldades relacionavam-se com a identificação dos alérgenos alimentares apresentados por meio de terminologias técnicas, a declaração de muitos ingredientes com termos genéricos que não identificam sua origem e também pela ausência de declaração de alguns constituintes alérgicos importantes na lista de ingredientes. Também foram debatidos a localização, contraste e o tamanho dos caracteres como obstáculos enfrentados pelos consumidores. Desta maneira, esta campanha foi uma das protagonistas no reconhecimento da nova rotulagem, que foi debatido com a Anvisa, entidades médicas, associações da indústria e de direitos do consumidor. ${ }^{11,15,19}$

Nesse seguimento, estudos também mostraram a dificuldade do consumidor em compreender as informações nos rótulos dos produtos alergênicos. Na pesquisa realizada por Binsfeld et al., ${ }^{20}$ observou-se que 39,5\% das reações alérgicas ao leite de vaca estavam relacionadas a erros na leitura dos rótulos pelos consumidores. Esse estudo demonstrou a importância que uma rotulagem adequada, contendo informações sobre a presença de alérgenos, poderia auxiliar na profilaxia de reações alérgicas.

Weber et al. ${ }^{21}$ avaliaram o desempenho de pais de crianças em dieta de exclusão do leite de vaca na identificação de alimentos industrializados com e sem leite de vaca. Os autores observaram que os termos iniciados com a palavra "leite" foram os mais reconhecidos. Entretanto, termos como "caseína", "caseinato" e "lactoalbumina" foram identificados por menos de $25 \%$ dos entrevistados, ressaltando o desconhecimento de termos técnicos pela população.

Outro estudo investigou a precisão da leitura de rótulos por pais de crianças alérgicas e verificou que a maioria dos entrevistados foi incapaz de reconhecer ingredientes alérgenos nos produtos, especialmente no leite de vaca e soja. ${ }^{22}$ 
É importante mencionar que os estudos nacionais que abordaram a verificação da rotulagem de alergênicos referente à nova resolução ainda são escassos. Até o momento, o estudo realizado por Freitas \& Piletti ${ }^{15}$ investigou a rotulagem de 15 produtos lácteos de diferentes marcas sob os critérios da RDC n. - 26/2015. Os autores verificaram que apenas três produtos informaram a presença de alergênicos, mas não mencionaram o período da realização do estudo, tampouco avaliaram as adequações em relação aos requisitos estabelecidos na legislação vigente. Sendo assim, vale ressaltar a relevância da presente investigação para a saúde pública.

No âmbito internacional, também foram verificados estudos que investigaram a declaração de alergênicos nos rótulos dos produtos. O estudo argentino realizado por López et al. ${ }^{23}$ verificou a declaração de alérgenos alimentares em 21 rótulos de alimentos comumente consumidos por crianças nos anos de 2012 e 2014, com o intuito de observar como as empresas têm feito a declaração de alérgenos nestes rótulos e as mudanças observadas em dois anos. Os autores observaram que foram acrescentados vários alérgenos nas frases de advertência, como também a presença de declaração em produtos que não o faziam anteriormente. Porém, em ambos os anos avaliados, foram encontradas inconsistencias, como a omissão da declaração "contém derivados de soja" em alimentos que possuíam lecitina de soja em sua composição, e a declaração de "contém" em alérgenos que não estavam presentes na lista de ingredientes. Vale ressaltar que, nesse país, a rotulagem de alimentos alergênicos não era obrigatória no período em que o estudo foi realizado.

Já a rotulagem preventiva, responsável por informar a presença de alérgenos em casos de contaminação cruzada, foi investigada por Zurzolo et al. ${ }^{24}$ nas embalagens de 1.355 produtos. Os autores australianos observaram que $65 \%$ dos produtos apresentaram a declaração de precaução para um ou mais alérgenos, e que os ingredientes mais listados na rotulagem preventiva foram nozes $(36,2 \%)$ e amendoim $(34,1 \%)$, seguidos de gergelim $(27,5 \%)$ e ovo $(22,6 \%)$. O estudo americano de Pieretti et al. ${ }^{25}$ determinou a frequência e a linguagem preventiva utilizada nos rótulos e identificou ambiguidades para os consumidores alérgicos a alimentos. Dos 20.241 produtos avaliados, apenas $17 \%$ continham informações preventivas nos rótulos. Vale ressaltar que nesses países a rotulagem preventiva ainda é opcional, e que na atual resolução brasileira, tal informação se destaca como requisito obrigatório.

Nesse contexto, a precisão na rotulagem de alergênicos é um meio para prevenir reações de hipersensibilidade a alimentos, uma vez que transmite informações extremamente importantes para os consumidores alérgicos que contam com a integridade e clareza da declaração de ingredientes alergênicos nas embalagens dos alimentos. ${ }^{6}$ As inadequações apresentadas neste estudo podem levar a sérios danos à saúde do consumidor alérgico, o que pode contribuir para onerar os gastos nos serviços públicos de saúde. E ainda, a fiscalização pelos órgãos regulamentadores é indispensável em relação ao cumprimento das exigências da rotulagem de alergênicos, a fim de prevenir possíveis reações adversas aos indivíduos alérgicos. 


\section{Conclusão}

A presente investigação mostrou que uma parcela das indústrias alimentícias ainda descumpre as exigências da Resolução n.o 26, de julho de 2015, uma vez que foram detectadas inadequações nos rótulos dos alimentos alergênicos avaliados. Vale ressaltar a relevância deste contexto para a saúde pública, uma vez que os consumidores alérgicos requerem maior cuidado e atenção antes do consumo de qualquer produto, com o intuito de evitar reações indesejáveis. A missão de fiscalização contínua e eficaz do cumprimento das exigências poderia ser um quesito a ser reavaliado pela ANVISA e demais órgãos responsáveis, com o objetivo de assegurar que o consumidor tenha acesso a informes seguros sobre os produtos.

\section{Colaboradoras}

Miranda CCS participou da concepção e planejamento do estudo, obtenção, análise e interpretação dos dados e redação do artigo; Gama LLA participou da concepção e planejamento do estudo, análise e interpretação dos dados, redação e revisão crítica da versão final do artigo.

Conflito de Interesses: Os autores declaram não haver conflito de interesses.

\section{Referências}

1. Agência Nacional de Vigilância Sanitária. Ministério da Saúde. Rotulagem nutricional obrigatória: manual de orientação aos consumidores. Brasília: ANVISA, UnB; 2008. 17 p.

2. Brasil. Lei no 9.782, 26 de janeiro de 1999. Define o Sistema Nacional de Vigilância Sanitária, cria a Agência Nacional de Vigilância Sanitária e dá outras providências. Diário Oficial da União. 27 jan. 1999.

3. Brasil. Agência Nacional de Vigilância Sanitária. Resolução RDC n. 259 de 20 de setembro de 2002: Regulamento Técnico sobre Rotulagem de Alimentos Embalados. Diário Oficial da União. 22 set. 2002.

4. Câmara MCC, Marinho CLC, Guilan MC, Braga AMCB. A produção acadêmica sobre a rotulagem de alimentos no Brasil. Rev Panam Salud Publica. 2008; 23(1):52-58.

5. Marins BR, Jacob SC, Peres F. Avaliação qualitativa do hábito de leitura e entendimento: recepção das informações de produtos alimentícios. Ciênc Tecnol Aliment. 2008; 28(3):579-585.

6. Taylor SL, Hefle SL. Ingredient and labeling issues associated with allergenic foods. Allergy. 2001; 56 Suppl 67:64-69.

7. Chaddad MCC. Informação sobre a presença de alérgenos nos rótulos de alimentos: responsabilidade do estado na garantia dos direitos à saúde e à alimentação adequada da população com alergia alimentar. Demetra - Alimentação, Nutrição e Saúde. 2014; 9:369-392. 
8. Ferreira S, Pinto M, Carvalho P, Gonçalves JP, Lima R, Pereira F. Alergia às proteínas do leite de vaca com manifestações gastrointestinais. Nascer e Crescer - Revista de Pediatria do Centro Hospitalar do Porto. 2014; 23(2):72-79.

9. Alcocer MJC, Ares SC, López-Calleja I. Recent advances in food allergy. Braz J Food Technol. 2016; 19:261-277.

10. Rosa S, Ribeiro F, Pinto PL. Ingestão dos alimentos cozinhados na alergia alimentar ao leite de vaca e ao ovo. Revista Portuguesa de Imunoalergologia. 2016; 24(1):9-24.

11. Associação Brasileira de Alergia e Imunologia [Internet]. [Acesso em: 19 set. 2016]. Disponível em: http://www.asbai.org.br/secao.asp?s=106\&id=950

12. Agência Nacional de Vigilância Sanitária. Perguntas e Respostas sobre Rotulagem de Alimentos Alergênicos. Gerência de avaliação de risco e eficácia para alegações. Gerência geral de alimentos. Brasília: ANVISA; 2015. 27 p.

13. Costa J, Oliveira MBPP, Mafra I. Alergénios alimentares: o que são, o que provocam e como detetálos? Química - Boletim da Sociedade Portuguesa de Química (SPQ). 2012; 127:33-38.

14. Brasil. Agência Nacional de Vigilância Sanitária. Resolução RDC n. 26 de 02 de julho de 2015: Requisitos para rotulagem obrigatória dos principais alimentos que causam alergias alimentares. Diário Oficial da União. 3 jul. 2015.

15. Freitas AR, Piletti R. Análise da rotulagem de produtos lácteos de diferentes marcas de acordo com a Legislação RDC n 26, de Julho de 2015. Revista de Ciências Agroveterinárias e Alimentos. 2016; (1). Disponível em: http://revista.faifaculdades.edu.br/index.php/cava/article/view/194/89

16. Speridião PGL, Morais MB. Intolerância à lactose e alergia alimentar. In: Cuppari L. Guias de medicina ambulatorial e hospitalar da EPM_UNIFESP. Guia de Nutrição: clínica no adulto. 3. ed. São Paulo: Manole; 2014. p. 476-476.

17. Jardim FBB, Farias GA, Dias LCFC, Afonso ALT. Rotulagem de alimentos: avaliação e orientação às indústrias e aos consumidores quanto aos aspectos legais e informativos dos rótulos. Boletim Técnico Instituto Federal de Educação, Ciência e Tecnologia - Triângulo Mineiro. 2016; 2(1):26-29.

18. Garcia PPC, Carvalho LPS. Análise da rotulagem nutricional de alimentos diet e light. Ensaios e Ciência: Ciências Biológicas, Agrárias e da Saúde. 2011; 15(4):89-103.

19. Brasil. Ministério da Saúde. Agência Nacional de Vigilância Sanitária. Consulta Pública n. o 29/2014. Proposta de Resolução de Diretoria Colegiada que dispõe sobre Rotulagem de Alergênicos em Alimentos. Gerência Geral de Alimentos, 2013. [acesso em: 12 out. 2016]. Disponível em: http:// www.idec.org.br/uploads/audiencias_documentos/anexos/Justificativa.pdf

20. Binsfeld BL, Pastorino AC, Castro APBM, Yonamine GH, Gushken AKF, Jacob CMA. Conhecimento da rotulagem de produtos industrializados por familiares de pacientes com alergia a leite de vaca. Rev. Paul Pediatr. 2009; 27(3):296-302.

21. Weber TK, Speridião PGL, Sdepanian VL, Fagundes Neto U, Morais MB. The performance of parents of children receiving cow's milk free diets at identification of commercial food products with and without cow's milk. J Pediatr. 2007; 83(5):459-464. 
22. Joshi P, Mofidi S, Sicherer SH. Interpretation of commercial food ingredient labels by parents of food-allergic children. J Allergy Clin Immunol. 2002; 109(6):1019-1021.

23. López LB, Martín ME, Ferrer PAR. Declaración de alérgenos alimentarios en rótulos de alimentos habitualmente consumidos por niños. Diaeta. Buenos Aires. 2016; 155(34):15-24.

24. Zurzolo GA, Mathai ML, Koplin JJ, Allen KK. Precautionary allergen labelling following new labelling practice in Australia. J Paediatr Child Health. 2013; 49(4):306-310.

25. Pieretti MM, Chung D, Pacenza R, Slotkin T, Sicherer SH. Audit of manufactured products: use of allergen advisory labels and identification of labeling ambiguities. J Allergy Clin Immunol. 2009; 124(4):337-34

Recebido: 22 de fevereiro, 2018

Revisado: 03 de julho, 2018

Aceito: 10 de agosto, 2018 
